\title{
Gene expression profiles of thymic neuroendocrine tumors (carcinoids) with ectopic ACTH syndrome reveal novel molecular mechanism
}

\author{
Yu-fang Bi ${ }^{1 *}$, Rui-xin Liu ${ }^{1 *}$, Lei Ye ${ }^{1}$, Hai Fang ${ }^{2}$, Xiao-ying $\mathrm{Li}^{1,3}$, Wei-qing Wang ${ }^{1}$, \\ Ji Zhang ${ }^{2}$, Kan-Kan Wang', Lei Jiang ${ }^{1}$, Ting-wei Su' ${ }^{1}$, Zhong-yuan Chen ${ }^{4}$ \\ and Guang Ning ${ }^{1,3}$
}

\begin{abstract}
${ }^{1}$ Department of Endocrinology and Metabolism, Shanghai Institute of Endocrine and Metabolic Diseases, Shanghai Clinical Center for Endocrine and Metabolic Diseases, ${ }^{2}$ State Key Laboratory of Medical Genomics, ${ }^{3}$ Shanghai Key Laboratory for Endocrine Tumors and ${ }^{4}$ Department of Thoracic Surgery, Ruijin Hospital Affiliated to Shanghai Jiao-Tong University School of Medicine, 197 Ruijin 2nd Road, Shanghai 200025, People's Republic of China

(Correspondence should be addressed to G Ning at Department of Endocrinology and Metabolism, Shanghai Institute of Endocrine and Metabolic Diseases, Shanghai Clinical Center for Endocrine and Metabolic Diseases, Ruijin Hospital Affiliated to Shanghai Jiao-Tong University School of Medicine, 197 Ruijin 2nd Road, Shanghai 200025, People's Republic of China;

Email: guangning@medmail.com.cn)

${ }^{*}(\mathrm{Y}-\mathrm{f} \mathrm{Bi}$ and $\mathrm{R}-\mathrm{x}$ Liu contributed equally to this work)
\end{abstract}

\begin{abstract}
Although there has been increased knowledge about the molecular biology of neuroendocrine tumors (NETs), little is known about thymic carcinoids and even less about those with excessive hormone disorders, such as ectopic ACTH syndrome. This study was designed to gain insights into the molecular networks underlying the tumorigenesis of thymic carcinoids with ACTH secretion. By an approach integrating cDNA microarray and methods of computational biology, we compare gene expression profile between ACTH-producing thymic carcinoids and the normal thymus. In total, there are 63 biological categories increased and 108 decreased in thymic carcinoids. Cell proliferation was stimulated, which may explain the relatively uncontrolled cell growth of the tumor. Dysregulation of the Notch-signaling pathway was likely to be underlying the neuroendocrine features of this type of tumors. Moreover, inhibition of immunity and increased neuropeptide signaling molecules (POMC and its sorting molecule CPE) made the clinical manifestation reasonable and thus validated the array data. In conclusion, thymic carcinoids have a distinct gene expression pattern from the normal thymus, and they are characterized by deregulations of a series of biofunctions, which may be involved in the development of NETs. Hence, this study has provided not only a detailed comprehension of the molecular pathogenesis of thymic carcinoids with ectopic ACTH syndrome, but also a road map to approach thymic NETs at the system level.
\end{abstract}

Endocrine-Related Cancer (2009) 16 1273-1282

\section{Introduction}

Carcinoid tumors were first described as multiple tumors in the distal ileum at autopsy (Lubarsch 1888). The term 'karzinoide' was then used to describe a group of tumors that behaved in a more indolent fashion than typical adenocarcinomas. Carcinoid tumors derived from the thymus were first documented in 1972 (Rosai \& Higa 1972). It can be associated with ectopic ACTH syndrome or may be a component of multiple endocrine neoplasia syndrome type 1 (MEN1). Most carcinoids are benign in behavior, whereas thymic carcinoids display a much more 
aggressive phenotype than those originating from other locations (Duh et al. 1987, Moran \& Suster 2000, Gal et al. 2001, Kondo \& Monden 2003, Tiffet et al. 2003). For the thymic carcinoids with ectopic ACTH syndrome, excessive ACTH production makes the tumors even more aggressive.

As to the potential molecular mechanism, efforts have been focused on the understanding of ectopic ACTH production, while the knowledge about the tumorigenesis is limited and most are genetic studies. There is one study which revealed chromosomal imbalances in ten neuroendocrine thymic tumors, including gains on chromosome Xp, 7p, 7q, 11q, 12q and 20q, and losses at 6q, 6p, 4q, 3p, 10q, 11q and 13q (Rieker et al. 2005). Loss of heterozygosity (LOH) at chromosome $1 \mathrm{p}$ has been reported in two thymic neuroendocrine tumors (NETs; Teh et al. 1998). Loss of chromosomes 3, 9p21-pter, $\mathrm{Y}$ and gain of chromosome $19 \mathrm{p}$ were discovered in one case (Leotlela et al. 2003). Although 25\% of the reported thymic NETs are from MEN1 patients (Teh et al. 1998), LOH of the MEN1 locus on chromosome 11q13 has not been reported in thymic NETs except in one patient. However, no study has yet been performed at the transcriptome level in thymic carcinoids.

To get insights into the molecular pathways responsible for the tumorigenesis of this type of neuroendocrine neoplasm, we conducted cDNA microarrays in five thymic carcinoids with ectopic ACTH secretion and six normal thymus controls.

\section{Materials and methods}

\section{Patients}

The study was approved by the Ruijin Hospital Ethics Committee for Human Research. Informed consent was obtained from each subject participating in the study after a full explanation of the purpose and nature of all procedures used. Five ACTH-producing human thymic carcinoid (ACs) tissues were obtained at surgery from patients with ectopic ACTH syndrome (Table 1), and AC5 was the recurrent tumor of AC4. The patients presented with a typical Cushing habitus accompanied by hyperpigmentation and hypokalemia. For all these four patients, the high-dose $(8 \mathrm{mg})$ dexamethasone suppression test showed lack of suppression, computed tomography scanning documented anterior mediastinal masses, and the removed mediastinal tumors were confirmed as ACs by positive ACTH and NSE staining (Wang et al. 2006). The six noncancerous thymuses (NCs) were from independent individuals without ectopic ACTH syndrome.

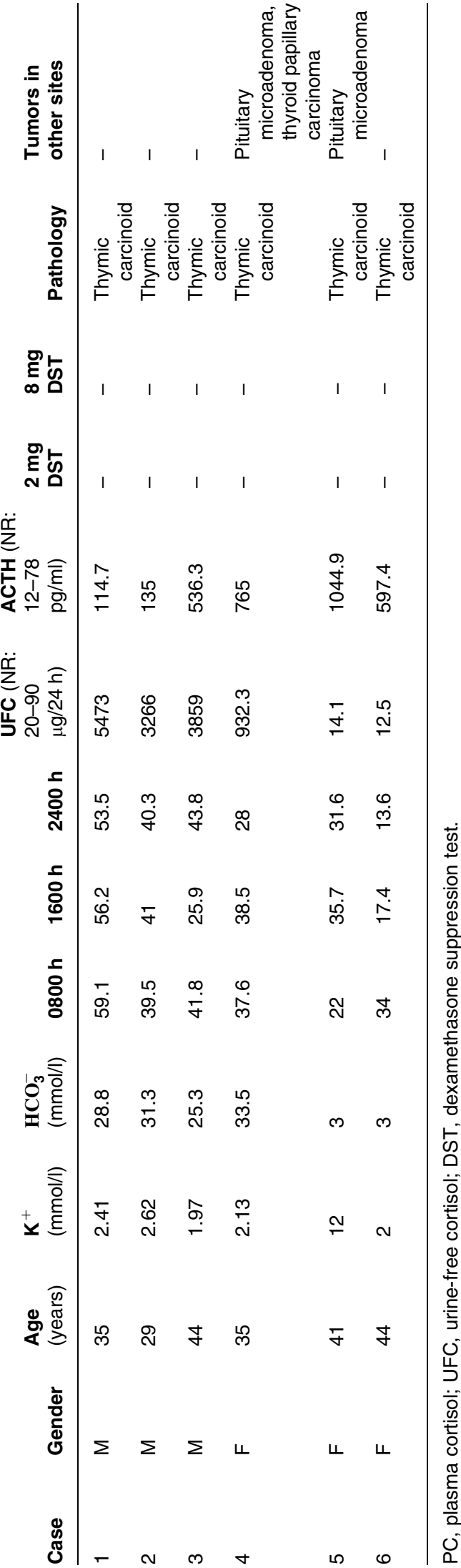


While we were preparing this manuscript, two additional ACTH-producing human thymic carcinoids from patients with ectopic ACTH syndrome were obtained (AC6 and AC7).

\section{cDNA microarray}

Total RNA was prepared using TRIzol (Life Technologies, Inc.), further purified with RNeasy column (Qiagen). For each of the 11 specimens, $\sim 30 \mu \mathrm{g}$ RNA was reversely transcribed into cDNA primed with oligo (dT) and labeled with Cy5-dCTP (Red fluorescent dye, R) using Superscript II reverse transcriptase (Life Technologies, Inc.), while the reference used in all hybridizations was prepared by pooling cDNA from six NCs and labeled with Cy3-dCTP (Green fluorescent dye, G). Microarrays with 12630 cDNA clones representing 10647 genes were fabricated in-house using a Generation III spotter (Amersham Biosciences). The cDNA clones were sequence verified and enriched with genes expressed in hematopoietic cells (Mao et al. 1998, Zhang et al. 2000). Among these, cDNA clones include commercial clones from Invitrogen. The scorecard plate including positive control, negative control, dynamic range control, ratio control, and housekeeping genes was spotted on each slide. Detailed information can be found at http://www1.amershambiosciences.com/ aptrix/upp01077.nsf/Content/Products?OpenDocument\&parentid $=63004285 \&$ moduleid $=165076 \#$ content. Microarray slides were obtained from Full moon BioSystems (Sunnyvale, CA, USA). The clones were spotted in a final concentration of $200-400 \mathrm{fmol} / \mu \mathrm{l}$ in spotting buffer (50\% DMSO) using 12 microspot pins to reach a complexity of 12630 spots per slide. After spotting, the slides were u.v. cross-linked $(400 \mathrm{~mJ})$ and stored at room temperature (Zheng et al. 2005, Du et al. 2006).

\section{Data mining}

After normalization by intensity-dependent global LOWESS regression for each array, only those cDNAs whose expression was detected in at least $50 \%$ of both ACs and NCs were included for statistical analysis. After such preprocessing, 7081 well-measured cDNAs remained for further analysis. The preprocessed data sets were subjected to significance analysis of microarrays (SAM; Tusher et al. 2001) by using the unpaired two-class comparison for identifying differentially expressed cDNAs between $\mathrm{NCs}$ ( $\mathrm{NC} 1, \mathrm{NC} 2, \mathrm{NC} 3$, NC4, NC5, and NC6) and ACs (AC1, AC2, AC3, AC4, and AC5). SAM utilizes a modified $t$-test statistic and sample-labeled permutations to evaluate statistical significance measured by the false discovery rate (FDR; Storey \& Tibshirani 2003), an estimate of the fraction of falsely significant genes. A significance threshold was expected to be at least 1.5 -fold changes and FDR of 1\%. Under such a threshold, 2409 significant cDNAs were identified. Furthermore, fold changes in transcript levels were calculated from the mean $\log 2$ expression values of ACs group versus the mean of NCs group, and factitiously reviewed as additive specimen labeled as 'FC'.

As for gene clustering and visualization, a self organising map (SOM) software package implemented with the Matlab 6.5 environment was utilized to train log-transformed (base 2) ratios of intensities between 2409 significant cDNAs over 11 specimens as well as FC with eighty-one $(9 \times 9)$ neurons. Illustration of the SOM outputs was visualized by component plane presentation (CPP; Xiao et al. 2003, Fang et al. 2008), each presentation illustrating a sample-specific, genome-wide transcriptional map. The SOM outputs by CPP revealed distinct transcriptome profiles between two phenotypes, NCs versus ACs. Such profiling features might provide insights into the biology of thymic carcinoids with ectopic ACTH syndrome.

The search for enriched gene ontology (GO) functional categories in the lists of differentially expressed genes was conducted with MAPPFinder 2.0. The permuted $P$ value was calculated by MAPPFinder 2.0 as a statistical measure of significance for gene expression in a given GO functional category. Output from the MAPPFinder was manually filtered to remove processes that represented the same genes (typically parent-child processes).

\section{Real-time PCR}

The SYBR Green assay contained $5 \mu \mathrm{l} 2 \times$ SYBR Green Master Mix buffer (PE Biosystems, Warrington, $\mathrm{UK}), 0.1 \mu \mathrm{l}$ forward and $0.1 \mu \mathrm{l}$ reverse primer (20 mM), $1 \mu \mathrm{l} \mathrm{cDNA}$, and $3.8 \mu \mathrm{l} \mathrm{ddH}_{2} \mathrm{O}$. PCR was carried out by a ABI PRISM 7900 system (PerkinElmer, Foster City, CA, USA) as follows: one cycle of $95^{\circ} \mathrm{C}$ for $10 \mathrm{~min}$ (hot start) and 40 cycles of three steps $\left(95^{\circ} \mathrm{C}\right.$ for $30 \mathrm{~s}, 59^{\circ} \mathrm{C}$ for $30 \mathrm{~s}$, and $72{ }^{\circ} \mathrm{C}$ for $\left.30 \mathrm{~s}\right)$. At the end of the amplification, a dissociation curve (melting curve) was built in the temperature range of 65-95 ${ }^{\circ} \mathrm{C}$. All amplifications and detections were carried out in a MicroAmp optical 96-well reaction plate with optical adhesive covers (Applied Biosystems, Foster City, CA, USA). PCRs were performed in triplicate, and $\beta$-actin was coamplified to normalize the amount of RNA added to the reaction. All data were analyzed using the ABI PRISM SDS 2.0 software. 

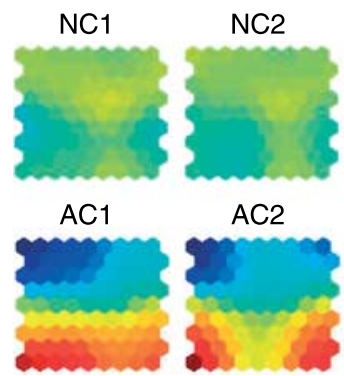

AC2

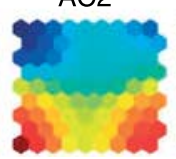

NC3

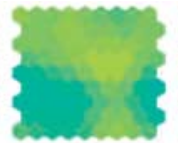

AC3

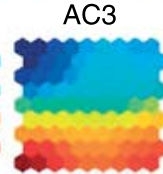

NC4

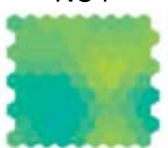

AC4

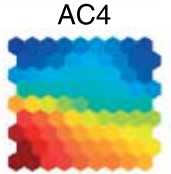

NC5

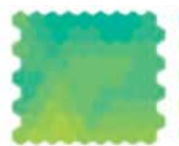

AC5

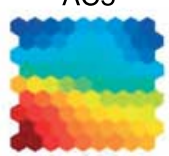

NC6

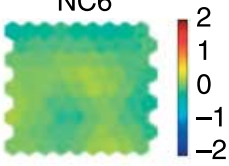

FC

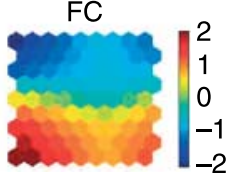

Figure 1 Component plane presentation-integrated self-organizing map (CPP-SOM) of thymic neuroendocrine tumors (carcinoids) with ectopic ACTH syndrome. Eighty-one $(9 \times 9)$ neurons were utilized to train log-transformed (base 2) ratios of intensities 2409 significant cDNAs over 11 specimens as well as 'FC'. Each presentation illustrates a sample-specific, genomewide transcriptional map in which up-regulated (red), down-regulated (blue), and moderately regulated (yellow and green) are well delineated. Color-coding index stands for log2 ratios, with the brighter color to denote the higher value. Noncancerous thymus (NCs) and ACTH-secreting thymic carcinoid (ACs) displayed homologous transcriptome pattern respectively, while distinct between the two groups. FC factitiously represented the expression differences between mean ACs group versus the mean of NCs group.

\section{Results}

\section{Transcriptome profiles of thymic NETs (carcinoids) with ectopic ACTH syndrome}

To analyze the mechanisms underlying thymic carcinoids with ectopic ACTH syndrome, we performed transcriptome profiling on five samples (ACs) from thymic tumor patients with ectopic ACTH syndrome (Table 1) and six samples (NCs) of the NCs. After microarray hybridization and data acquisition, gene expression data were subjected to SAM to determine those genes with statistically significant differences. By using the criteria of FDR $<1 \%$ and at least 1.5-fold changes, 2409 cDNAs were selected representing the transcriptome signatures of thymic carcinoids with ectopic ACTH syndrome. We then applied CPP-integrated SOM (CPP-SOM) for gene clustering and visualization of significant expression data. As shown in Fig. 1, CPP-SOM offers a global view of gene clustering, particularly with respect to the expression patterns. Genes mapped to the corner/edge areas of the map are mostly regulated, with red representing up-regulation and blue representing down-regulation. Also, each presentation of SOM illustrates a sample-specific transcriptome map, permitting direct comparisons of transcriptome differences between two phenotypes NCs versus ACs. Furthermore, gene expression-based sample relationships were visualized in the three-dimensional space captured by principal component analysis, as demonstrated in Fig. 2. Both of these analyses revealed homologous transcriptome profiles within NCs (and $\mathrm{ACs}$ ), while distinct transcriptome profiles between NCs and ACs. It is of note that AC4 and AC5 shared a similar expression pattern (Fig. 1) and the closer sample relationship (Fig. 2) when compared with other samples of ACs. It was consistent with the factor that AC5 was the recurrence of AC4, and also indicated that similar biological behavior could be reflected by similar expression profile.

To characterize the major biological processes $(\mathrm{P})$, molecular functions $(\mathrm{F})$, and cellular components $(\mathrm{C})$, we used MAPPFinder (a component of GenMAPP version 2.0) to link gene expression data to the GO hierarchy. MAPPFinder produced a statistical list (based on permutated $P$ value $<0.05$ ) of GO biological categories associated with the differentially

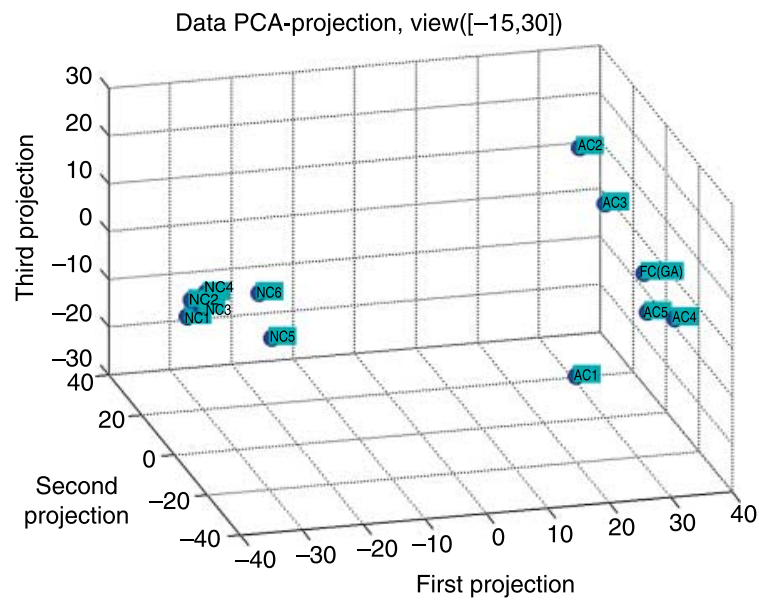

Figure 2 Visualization of sample relationships by principal component analysis (PCA). The three-dimensional plot illustrates PCA of 11 samples as well as FC illustrating the relationships based on 2409 significant cDNAs expression data. $\mathrm{NC}$, noncancerous thymus; AC, ACTH-secreting thymic carcinoid. FC factitiously represented the expression differences between mean ACs group versus the mean of NCs group. 
expressed genes, from which the most significant nonsynonymous categories with at least three genes changed were identified (permutated $P<0.05$ ). In total, there are 63 biological categories increased and 108 decreased in thymic carcinoids. We then focused on those that might explain the tumor behavior and clinical presentations. After quantitative PCR confirming the aberrant expression of some of the potential candidate genes both in the four tumors using microarray data and two additional ACTH-producing thymic carcinoids (Fig. 3), an overview of the molecular pathology involvement was revealed and was addressed as follows.

\section{Stimulation of cell proliferation pathway coordinating tumor phenotype}

GO analysis revealed significantly increased regulation of cell proliferation. Table 2 demonstrated all the genes related to proliferation and those significantly deregulated in thymic carcinoids, with $C D C 25 B$ the most up-regulated, $C T B P 1$ the most down-regulated, and also the deregulated $\mathrm{Wnt} / \beta$-catenin-signaling pathway (Fig. 3A and B).

\section{Wnt and Notch pathways coordinating the characteristics of neuroendocrine differentiation}

Among the aberrant expression of genes involved in cell differentiation, the Wnt-signaling pathway was revealed as being tightly associated with thymic carcinoids (Table 3). CTNNB1, as previously described, was present at higher levels in AC patients. The expression of $\beta$-catenin target genes, $M Y C(c-M y c)$ and CCND1 (cyclin D1), was also examined in AC tissues. CCND1 expression was consistently high in $\mathrm{AC}$ tumors and $c-M y c$ expression changed modestly (Fig. 3B). $P P P 2 C B$, which was up-regulated on average about three times (Fig. 3C), encodes the phosphatase 2A (PP2A) catalytic subunit. Consistently lower expression of $\mathrm{NOTCH} 2$, the encoding gene for one of the Notch family members, was observed in our thymic carcinoid compared with the normal thymus (Fig. 3C).

\section{Molecular pathways coordinating the clinical manifestation of thymic carcinoids with ectopic ACTH syndrome}

Unlike the etiological pathways described above, some other pathways revealed in our data might be a consequence of the tumors. The up-regulated
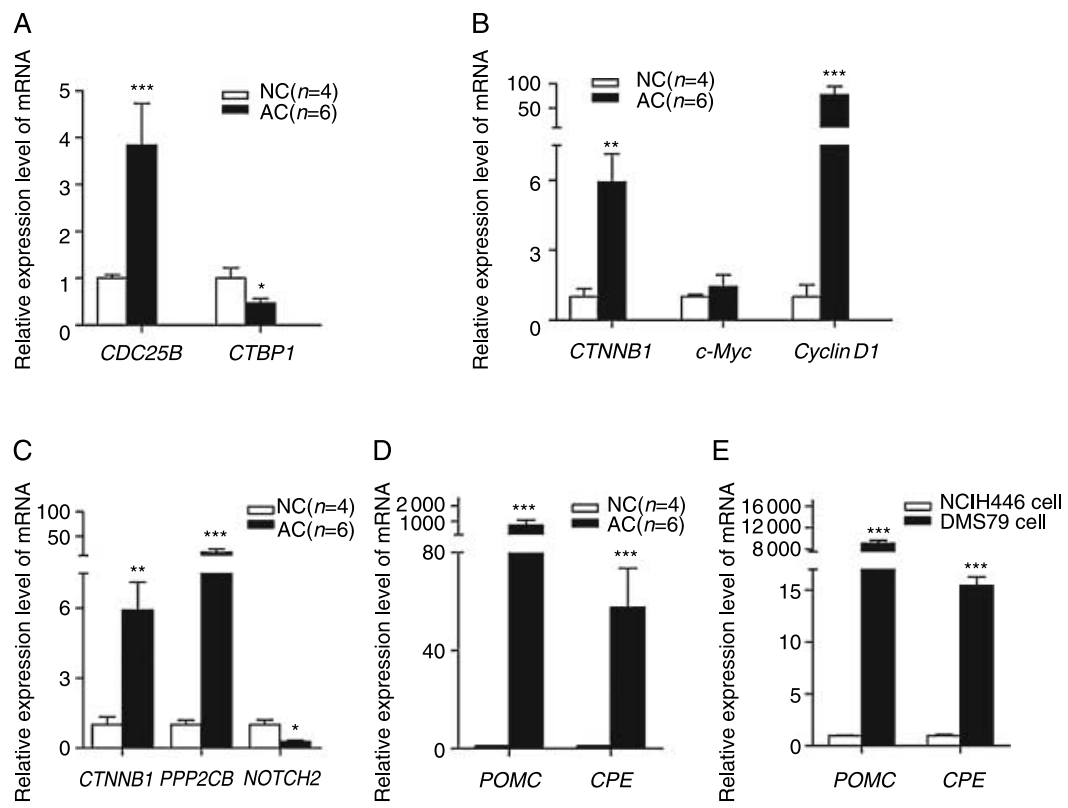

Figure 3 Quantitative PCR revealed consistent alteration with the array data in the thymic carcinoid tissues. (A) Increased expression of $C D C 25 B$ and decreased expression of $C T B P 1$ in AC patients $(n=6)$ compared with NC patients $(n=4)$. (B) $\beta$-Catenin and its downstream target genes expression was up-regulated in AC patients $(n=6)$. (C) Decreased expression of NOTCH2 and Wnt pathway molecules in AC patients $(n=6)$. (D) POMC and CPE gene expression increased dramatically in AC patients $(n=6)$. (E) POMC and CPE displayed much higher expression level on DMS79 cell compared with NCIH446 cell. Gene expression level was normalized to the corresponding levels of $\beta$-actin mRNA. Data are represented as the mean \pm S.E.M. Unpaired Student's $t$-test was used for evaluation of statistical significance. ${ }^{\star} P<0.05$, ${ }^{\star \star} P<0.01$, and ${ }^{\star \star \star} P<0.001$. NC, noncancerous thymus; AC, ACTH-secreting thymic carcinoid. 
$Y-f B i, R-x$ Liu et al.: Gene expression profiles of thymic carcinoids

Table 2 Enriched functional category of genes involved in regulation of cell proliferation

\begin{tabular}{|c|c|c|c|c|c|c|c|c|}
\hline Genbank & Symbol & FC & AC1 & AC2 & AC3 & AC4 & AC5 & FDR (\%) \\
\hline NM_021874 & $C D C 25 B$ & 3.73 & 2.92 & 4.55 & 5.40 & 1.47 & 3.34 & 0.02 \\
\hline NM_006454 & $M X D 4$ & 2.20 & 1.88 & 2.48 & 2.22 & 2.23 & 2.13 & 0.04 \\
\hline NM_003641 & IFITM1 & 1.71 & 1.31 & 1.83 & 1.73 & 1.64 & 1.51 & 0.03 \\
\hline NM_003029 & SHC1 & 1.62 & 1.79 & 0.50 & 1.39 & 1.16 & 1.26 & 0.03 \\
\hline NM_001904 & CTNNB1 & 1.54 & -0.36 & 2.49 & 0.41 & 1.29 & 1.86 & 0.29 \\
\hline NM_002032 & FTH1 & 1.49 & 1.95 & 1.39 & 1.29 & 1.43 & 1.31 & 0.03 \\
\hline AF018081 & COL 18A1 & 1.32 & -0.37 & -0.03 & 2.27 & 1.87 & 1.92 & 0.29 \\
\hline NM_005655 & TIEG & 1.09 & 0.94 & 0.65 & 0.78 & 1.55 & 1.21 & 0.20 \\
\hline NM_000508 & $F G A$ & 1.02 & 0.82 & 1.59 & 1.03 & 1.94 & 0.28 & 0.22 \\
\hline NM_002831 & PTPN6 & -0.97 & -0.40 & -0.69 & -1.13 & -0.96 & -1.00 & 0.24 \\
\hline AV654571 & $P C A F$ & -1.01 & -0.08 & -1.39 & -0.62 & -1.29 & -1.24 & 0.53 \\
\hline NM_004235 & $K L F 4$ & -1.13 & -0.70 & -0.73 & -1.14 & -1.38 & -0.73 & 0.44 \\
\hline NM_006019 & TCIRG1 & -1.14 & -1.13 & -0.25 & -0.90 & -1.32 & -1.05 & 0.46 \\
\hline NM_005620 & $S 100 A 11$ & -1.24 & -1.92 & -1.27 & -2.63 & -1.95 & -1.30 & 0.13 \\
\hline NM_016418 & $N F 2$ & -1.24 & -0.25 & -1.28 & -0.59 & -1.40 & -1.37 & 0.44 \\
\hline NM_003144 & SSR1 & -1.29 & -0.46 & -2.02 & -0.72 & -1.44 & -1.42 & 0.40 \\
\hline NM_005746 & PBEF1 & -1.32 & -1.73 & -0.76 & -1.12 & -1.50 & -1.41 & 0.41 \\
\hline NM_005962 & $M X I 1$ & -2.00 & -1.17 & -3.00 & -2.77 & -2.22 & -1.69 & 0.25 \\
\hline NM_001328 & CTBP1 & -3.38 & -1.65 & -4.80 & -6.03 & -2.27 & -2.13 & 0.09 \\
\hline
\end{tabular}

AC, ACTH-secreting thymic carcinoid.

neuropeptide-signaling pathway is among them (Table 4). POMC, the ACTH encoding gene, was strikingly up-regulated by more than four times in these patients. $C P E$ with the corresponding protein carboxypeptidase $\mathrm{E}$ was increased by more than three times (Fig. 3D). We also examined POMC and CPE expression in DMS79 and NCIH446 cell lines, which respectively represent human ACTH-producing small cell lung cancer and nonACTH-producing small cell lung cancer. In accordance with the results of the patients, DMS79 cell line displayed high levels of $P O M C$ and $C P E$ expression (Fig. 3E), which to some extent compensate with the display on the patients.

\section{Discussions}

As a type of neoplasm, thymic carcinoids are also characterized by uncontrolled cell proliferation, although with relatively benign features. CDC25B is a member of the CDC25 family of phosphatases, which primarily targets cyclin $\mathrm{B}-\mathrm{Cdk} 1$ and controls the entry into mitosis. Both in vitro and in vivo studies have confirmed an oncogenic role for CDC25 (Kristjansdottir \& Rudolph 2004, Boutros et al. 2007), especially the clear and consistent trend associating CDC25 with breast cancer (Galaktionov et al. 1995, Ma et al. 2001). As to those tumors with neuroendocrine origin, it was reported that only

Table 3 Enriched functional category of genes involved in regulation of cell differentiation

\begin{tabular}{|c|c|c|c|c|c|c|c|c|}
\hline Genebank & Symbol & FC & AC1 & AC2 & AC3 & AC4 & AC5 & FDR (\%) \\
\hline NM_004156 & РPP2CB & 2.64 & 2.24 & 1.53 & 2.84 & 2.87 & 2.70 & 0.01 \\
\hline D80010 & LPIN1 & 1.92 & 2.62 & 0.57 & 1.37 & 2.32 & 2.24 & 0.02 \\
\hline NM_014707 & HDAC9 & 1.86 & 1.75 & 2.16 & 1.85 & 1.53 & 1.74 & 0.01 \\
\hline NM_014863 & $\begin{array}{l}\text { GALNAC4S- } \\
\quad 6 S T\end{array}$ & 1.52 & 2.11 & 1.92 & 1.58 & 0.56 & 0.48 & 0.01 \\
\hline NM_012443 & SPAG6 & 1.34 & 1.04 & 1.00 & 0.60 & 1.64 & 1.56 & 0.01 \\
\hline NM_016645 & NEUGRIN & 1.31 & 1.58 & 1.49 & 1.62 & 0.51 & 0.73 & 0.01 \\
\hline NM_000214 & $J A G 1$ & 1.11 & 1.10 & -0.37 & 1.10 & 1.71 & 1.66 & 0.13 \\
\hline AB0 44088 & $B H L H B 3$ & 1.08 & 0.46 & 1.35 & 1.28 & 0.27 & 0.91 & 0.24 \\
\hline NM_001242 & TNFRSF7 & -1.11 & 0.12 & -1.31 & 0.02 & -0.92 & -0.81 & 0.24 \\
\hline AF208043 & IFI16 & -1.17 & 0.33 & -1.53 & -2.06 & -1.55 & -1.53 & 0.06 \\
\hline NM_021103 & TMSB10 & -1.31 & -2.52 & -0.44 & -0.90 & -1.39 & -1.39 & 0.01 \\
\hline AJ227863 & MBNL1 & -1.50 & -0.73 & -1.87 & -1.61 & -1.34 & -1.52 & 0.01 \\
\hline NM_024408 & NOTCH2 & -1.58 & -1.17 & -2.47 & -0.92 & -1.85 & -1.54 & 0.01 \\
\hline
\end{tabular}

AC, ACTH-secreting thymic carcinoid. 
Table 4 Enriched functional category of genes involved in regulation of neuropeptide signaling pathway

\begin{tabular}{|c|c|c|c|c|c|c|c|c|}
\hline Genebank & Symbol & FC & AC1 & $\mathrm{AC2}$ & AC3 & AC4 & AC5 & FDR (\%) \\
\hline NM_000939 & POMC & 4.27 & 2.92 & 4.55 & 5.40 & 1.47 & 3.34 & 0.06 \\
\hline NM_001873 & $C P E$ & 3.29 & 3.07 & 3.99 & 3.28 & 3.06 & 2.99 & 0.02 \\
\hline AL161973 & MLLT4 & 1.65 & 1.20 & 1.23 & 0.53 & 2.15 & 1.77 & 0.02 \\
\hline AF307080 & LPHN3 & 1.11 & 0.18 & 1.45 & 0.36 & 1.78 & 1.03 & 0.10 \\
\hline NM_012302 & LPHN2 & 0.99 & 0.81 & -0.23 & 0.02 & 0.40 & 1.04 & 0.10 \\
\hline AB018301 & GPR116 & 0.82 & 0.35 & 0.05 & 1.16 & 1.47 & 1.40 & 0.29 \\
\hline AF478567 & PDZGEF2 & -0.92 & -0.90 & -0.66 & -0.97 & -0.49 & -0.50 & 0.12 \\
\hline NM_016340 & PDZGEF2 & -0.94 & -0.32 & -0.69 & -0.69 & -0.39 & -0.37 & 0.08 \\
\hline $\mathrm{BC} 000316$ & $R A B 2 L$ & -1.00 & -0.76 & -0.72 & -0.92 & -1.10 & -1.49 & 0.12 \\
\hline NM_001703 & $B A I 2$ & -1.10 & -1.22 & -0.77 & -1.03 & -1.16 & -1.14 & 0.05 \\
\hline NM_004761 & $R A B 2 L$ & -1.11 & -1.39 & -0.86 & -1.06 & -0.86 & -1.05 & 0.05 \\
\hline NM_014737 & RASSF2 & -1.17 & -1.25 & -1.24 & -1.61 & -1.32 & -1.10 & 0.02 \\
\hline
\end{tabular}

AC, ACTH-secreting thymic carcinoid.

CDC25-positive medullary thyroid carcinoma showed a significantly worse disease-free survival rate than those without expression (Ito et al. 2005). CTBP1, which is the most down-regulated gene in our study of thymic carcinoids, encodes a phosphoprotein belongs to CTBP family. CTBP proteins are conserved among vertebrates as well as invertebrates and function as transcriptional corepressors. Possibly by binding and modulating E1A (Delouis et al. 2005), Evi-l oncogene (Senyuk et al. 2002), BRCA1 (Izutsu et al. 2001), and P53 (Paliwal et al. 2006), CTBPs are tightly involved in oncogenesis in humans. Loss of CTBP expression has been reported in malignant melanoma and knockout of wild-type $C T B P$ was associated with progression of human melanoma (Poser et al. 2002).

The canonical Wnt-signaling pathway has a critical role in cell fate determination (e.g. the decision to proliferate or differentiate; Reya \& Clevers 2005). $C T N N B 1 / \beta$-catenin ( $\beta$-catenin), which is the central and essential component in the Wnt-signaling cascade, was up-regulated more than five times in AC patients. Aberrant activation of $\beta$-catenin promotes cell proliferation and initiates colorectal tumorigenesis (van de Wetering et al. 2002). More recently, Kim et al. (2008) reported that of 51 solid pseudopapillary neoplasm cases, $94.4 \%$ were positive for nuclear $\beta$-catenin, which indicates that the $\mathrm{Wnt} / \beta$-catenin pathway might take part in NETs tumorigenesis. To search for more evidence of Wnt pathway activation, we examined the $\beta$-catenin target genes MYC $(c-M y c)$ and CCND1 (cyclin D1) expression in AC tissues. We found consistently high level of cyclin $D_{1}$ in AC tumors, which is required for cell cycle $\mathrm{G}_{1} / \mathrm{S}$ transition and affects cell cycle progression. However, $c-M y c$ expression changed only modestly. This difference may be attributed in part to tissue-specific characteristics of Wnt target genes. Besides these extremely deregulated genes, 11 other genes displayed increased expression and 10 decreased. It is then speculated that up-regulation of genes like $C D C 25 B, \mathrm{Wnt} / \beta$-catenin and down-regulation of genes like $C T B P$ indicated higher modulation of cell growth in thymic carcinoids.

Compared with common tumors derived from epithelial cells, thymic carcinoids display remarkable neuroendocrine differentiation. NETs are a very heterogeneous group arising from neuroendocrine cells, which are distributed in many tissues and organs. Considering the morphological and physiological similarity, it is most likely that there is a specific common genetic switch underlying this large group of specific tumors (Barakat et al. 2004), while little has been discovered yet. As we described above, the Wnt/ $\beta$-catenin pathway not only promotes cell proliferation but also affects cell differentiation. It has been implicated in neuroendocrine differentiation, such as endocrine cell development in the anterior lobe of pituitary (Treier et al. 1998) and neuroendocrine transdifferentiation of prostate cancer cells (Yang et al. 2005). Higher expression of CTNNB1 in AC patients indicated its potential participation in neuroendocrine differentiation of thymic carcinoids. Xenopus studies confirmed a positive role for the PP2A catalytic subunit in Wnt signal transduction (Ratcliffe et al. 2000). The Notch family is another evolutionarily conserved signaling pathway that controls cellular differentiation. NOTCH2, the encoding gene for one of the family members, is down-regulated in the differentiated corticotrope (Raetzman et al. 2004), and persistent expression of $\mathrm{NOTCH} 2$ could delay gonadotrope differentiation (Raetzman et al. 2006). The authors considered that the absence of Notch signaling may be important to permit differentiation, which might also be the case in our thymic carcinoids, since consistently lower expression was observed 
compared with the normal thymus. Although other evidence is necessary to establish the roles of Wnt and Notch pathways in the neuroendocrine features of thymic carcinoids, our data has provided a clue.

$C P E$ has been found to be involved in pulmonary NETs (He et al. 2004), pituitary adenomas (Fan et al. 2002), and insulinoma (Wang et al. 2004). Suppression of pathways involved in the immune response, inflammatory response, antigen processing, and immune cell activation explained the immunosuppression status of such patients.

From our data, it is not possible to reflect the aggressive biologic behavior of thymic carcinoids, because three out of four patients demonstrated benign features and are still well after follow-up for 5-6 years, and only one suffered recurrence 4 years after the first surgery. Consistently, the functional category associated with cell motility was down-regulated in thymic carcinoids.

Ectopic ACTH syndrome caused by nonpituitary NETs has been known for several decades, and numerous studies have been trying to clarify the molecular basis of POMC deregulation. This included large-scale gene expression analysis in bronchial carcinoids (Pascual-Le Tallec et al. 2002) and DMS-79 cells (Turney et al. 2004). To the best of our knowledge, this is the first study focusing on the tumorigenesis of this type of tumor.

In summary, thymic carcinoids have a distinct expression pattern compared with the normal thymus. They are characterized by deregulations of many biofunctions including abnormal proliferation and differentiation signals, which may be involved in the development of NETs. Other abnormalities like activation of neuropeptide signaling and inhibition of immune response might explain the hormone disorder and immunity defects evident in ectopic ACTH syndrome.

\section{Declaration of interest}

There is no conflict of interest that would prejudice the impartiality of the research reported. Any financial or potential conflict of interest is fully declared within the text of the article.

\section{Funding}

This work was supported by the National Natural Science Foundation of China (grant numbers. 30871203, 2008; 30725037 and 30771018, 2007); the project sponsored by the Scientific Research Foundation for the Returned Overseas Chinese Scholars from Shanghai Personnel Bureau.

\section{Acknowledgements}

We are indebted to the fellows and nurses of Shanghai Clinical Center for Endocrine and Metabolic Diseases for taking care of the patients included in this study. We greatly appreciate the patients for their support in this study.

\section{References}

Barakat MT, Meeran K \& Bloom SR 2004 Neuroendocrine tumours. Endocrine-Related Cancer 11 1-18.

Boutros R, Lobjois V \& Ducommun B 2007 CDC25 phosphatases in cancer cells: key players? Good targets? Nature Reviews. Cancer 7 495-507.

Delouis C, Prochasson P, Laithier M \& Brison O 2005 Use of adenoviral E1A protein to analyze K18 promoter deregulation in colon carcinoma cells discloses a role for CTBP1 and BRCA1. BMC Molecular Biology 68.

Du YZ, Wang KK, Fang H, Li JM, Xiao DK, Zheng PZ, Chen YL, Fan HY, Pan XL, Zhao CJ et al. 2006 Coordination of intrinsic, extrinsic, and endoplasmic reticulum-mediated apoptosis by imatinib mesylate combined with arsenic trioxide in chronic myeloid leukemia. Blood 107 1582-1590.

Duh QY, Hybarger CP, Geist R, Gamsu G, Goodman PC, Gooding GA \& Clark OH 1987 Carcinoids associated with multiple endocrine neoplasia syndromes. American Journal of Surgery 154 142-148.

Fan X, Olson SJ, Blevins LS, Allen GS \& Johnson MD 2002 Immunohistochemical localization of carboxypeptidases $\mathrm{D}, \mathrm{E}$, and $\mathrm{Z}$ in pituitary adenomas and normal human pituitary. Journal of Histochemistry and Cytochemistry 50 1509-1516.

Fang H, Wang KK \& Zhang J 2008 Transcriptome and proteome analyses of drug interactions with natural products. Current Drug Metabolism 9 1037-1047.

Gal AA, Kornstein MJ, Cohen C, Duarte IG, Miller JI \& Mansour KA 2001 Neuroendocrine tumors of the thymus: a clinicopathological and prognostic study. Annals of Thoracic Surgery 72 1179-1182.

Galaktionov K, Lee AK, Eckstein J, Draetta G, Meckler J, Loda M \& Beach D 1995 CDC25 phosphatases as potential human oncogenes. Science 269 1575-1577.

He P, Varticovski L, Bowman ED, Fukuoka J, Welsh JA, Miura K, Jen J, Gabrielson E, Brambilla E, Travis WD et al. 2004 Identification of carboxypeptidase $\mathrm{E}$ and gamma-glutamyl hydrolase as biomarkers for pulmonary neuroendocrine tumors by cDNA microarray. Human Pathology 35 1196-1209.

Ito Y, Yoshida H, Tomoda C, Uruno T, Takamura Y, Miya A, Kobayashi K, Matsuzuka F, Kuma K, Nakamura Y et al. 2005 Expression of CDC25B and CDC25A in medullary thyroid carcinoma: CDC25B expression level predicts a poor prognosis. Cancer Letters 229 291-297.

Izutsu K, Kurokawa M, Imai Y, Maki K, Mitani K \& Hirai H 2001 The corepressor CTBP interacts with Evi-1 to repress transforming growth factor beta signaling. Blood $972815-2822$. 
Kim M-J, Jang S-J \& Yu E 2008 Loss of E-cadherin and cytoplasmic-nuclear expression of b-catenin are the most useful immunoprofiles in the diagnosis of solid-pseudopapillary neoplasm of the pancreas. Human Pathology 39 251-258.

Kondo K \& Monden Y 2003 Lymphogenous and hematogenous metastasis of thymic epithelial tumors. Annals of Thoracic Surgery 76 1859-1864.

Kristjansdottir K \& Rudolph J 2004 CDC25 phosphatases and cancer. Chemistry and Biology 11 1043-1051.

Leotlela PD, Jauch A, Holtgreve-Grez H \& Thakker RV 2003 Genetics of neuroendocrine and carcinoid tumours. Endocrine-Related Cancer 10 437-450.

Lubarsch O 1888 Uber den pimaeren krebs des ileum nebst Be-. merkungen ueber das gleichzeitige Vorkommen von krebs. und Tuberculos. Virchows Archiv 11 280-317.

Ma ZQ, Liu Z, Ngan ES \& Tsai SY 2001 CDC25B functions as a novel coactivator for the steroid receptors. Molecular and Cellular Biology 21 8056-8167.

Mao M, Fu G, Wu JS, Zhang QH, Zhou J, Kan LX, Huang QH, He KL, Gu BW, Han ZG et al. 1998 Identification of genes expressed in human CD34(+) hematopoietic stem/progenitor cells by expressed sequence tags and efficient full-length cDNA cloning. PNAS 95 8175-8180.

Moran CA \& Suster S 2000 Neuroendocrine carcinomas (carcinoid tumor) of the thymus. A clinicopathologic analysis of 80 cases. American Journal of Clinical Pathology 114 100-110.

Paliwal S, Pande S, Kovi RC, Sharpless NE, Bardeesy N \& Grossman SR 2006 Targeting of C-terminal binding protein (CTBP) by ARF results in p53-independent apoptosis. Molecular and Cellular Biology 26 2360-2372.

Pascual-Le Tallec L, Dulmet E, Bertagna X \& de Keyzer Y 2002 Identification of genes associated with the corticotroph phenotype in bronchial carcinoid tumors. Journal of Clinical Endocrinology and Metabolism 87 5015-5022.

Poser I, Golob M, Weidner M, Buettner R \& Bosserhoff AK 2002 Down-regulation of $\mathrm{COOH}$-terminal binding protein expression in malignant melanomas leads to induction of MIA expression. Cancer Research 62 5962-5966.

Raetzman LT, Ross SA, Cook S, Dunwoodie SL, Camper SA \& Thomas PQ 2004 Developmental regulation of Notch signaling genes in the embryonic pituitary: Prop1 deficiency affects Notch2 expression. Developmental Biology 265 329-3240.

Raetzman LT, Wheeler BS, Ross SA, Thomas PQ \& Camper SA 2006 Persistent expression of Notch2 delays gonadotrope differentiation. Molecular Endocrinology 20 2898-2908.

Ratcliffe MJ, Itoh K \& Sokol SY 2000 A positive role for the PP2A catalytic subunit in Wnt signal transduction. Journal of Biological Chemistry 275 35680-35683.

Reya T \& Clevers H 2005 Wnt signalling in stem cells and cancer. Nature 434 843-850.
Rieker RJ, Aulmann S, Penzel R, Schnabel PA, Blaeker H, Esposito I, Morresi-Hauf A, Otto HF, Hecker E, Dienemann $\mathrm{H}$ et al. 2005 Chromosomal imbalances in sporadic neuroendocrine tumours of the thymus. Cancer Letters 223 169-174.

Rosai J \& Higa E 1972 Mediastinal endocrine neoplasm, of probable thymic origin, related to carcinoid tumor. Clinicopathologic study of 8 cases. Cancer 29 1061-1074.

Senyuk V, Chakraborty S, Mikhail FM, Zhao R, Chi Y \& Nucifora G 2002 The leukemia-associated transcription repressor AML1/MDS1/EVI1 requires CTBP to induce abnormal growth and differentiation of murine hematopoietic cells. Oncogene 21 3232-3240.

Storey JD \& Tibshirani R 2003 Statistical significance for genomewide studies. PNAS 100 9440-9445.

Teh BT, Zedenius J, Kytola S, Skogseid B, Trotter J, Choplin H, Twigg S, Farnebo F, Giraud S, Cameron D et al. 1998 Thymic carcinoids in multiple endocrine neoplasia type 1. Annals of Surgery 228 99-105.

Tiffet O, Nicholson AG, Ladas G, Sheppard MN \& Goldstraw P 2003 A clinicopathologic study of 12 neuroendocrine tumors arising in the thymus. Chest 124 141-146.

Treier M, Gleiberman AS, O'Connell SM, Szeto DP, McMahon JA, McMahon AP \& Rosenfeld MG 1998 Multistep signaling requirements for pituitary organogenesis in vivo. Genes and Development 12 1691-1704.

Turney MK, Nicholson WE \& Kovacs WJ 2004 Gene expression phenotyping of an ACTH-producing small cell lung cancer line. Molecular and Cellular Endocrinology 219 105-113.

Tusher VG, Tibshirani R \& Chu G 2001 Significance analysis of microarrays applied to the ionizing radiation response. PNAS 98 5116-5121.

Wang XC, Xu SY, Wu XY, Song HD, Mao YF, Fan HY, Yu F, Mou B, Gu YY, Xu LQ et al. 2004 Gene expression profiling in human insulinoma tissue: genes involved in the insulin secretion pathway and cloning of novel full-length cDNAs. Endocrine-Related Cancer 11 295-303.

Wang WQ, Ye L, Bi YF, Zhao HY, Sun SY, Tang ZY, Zhao YJ, Fang WQ, Chen ZY, Chen KM et al. 2006 Six cases of ectopic ACTH syndrome caused by thymic carcinoid. Journal of Endocrinological Investigation 29 293-297.

van de Wetering M, Sancho E, Verweij C, de Lau W, Oving I, Hurlstone A, van der Horn K, Batlle E, Coudreuse D, Haramis AP et al. 2002 The beta-catenin/TCF-4 complex imposes a crypt progenitor phenotype on colorectal cancer cells. Cell 111 241-250.

Xiao L, Wang KK, Teng Y \& Zhang J 2003 Component plane presentation integrated self-organizing map for microarray data analysis. FEBS Letters 538 117-124. 
Yang X, Chen MW, Terry S, Vacherot F, Chopin DK, Bemis DL, Kitajewski J, Benson MC, Guo Y \& Buttyan R 2005 A human- and male-specific protocadherin that acts through the wnt signaling pathway to induce neuroendocrine transdifferentiation of prostate cancer cells. Cancer Research 65 5263-5271.

Zhang QH, Ye M, Wu XY, Ren SX, Zhao M, Zhao CJ, Fu G, Shen Y, Fan HY, Lu G et al. 2000 Cloning and functional analysis of cDNAs with open reading frames for 300 previously undefined genes expressed in CD34+ hematopoietic stem/progenitor cells. Genome Research 10 1546-1560.

Zheng PZ, Wang KK, Zhang QY, Huang QH, Du YZ, Zhang QH, Xiao DK, Shen SH, Imbeaud S, Eveno E et al. 2005 Systems analysis of transcriptome and proteome in retinoic acid/arsenic trioxide-induced cell differentiation/apoptosis of promyelocytic leukemia. PNAS 102 7653-7658. 